\title{
On the origin of high Doppler velocity wings in the spectra of O-rich AGB stars
}

\author{
Do Thi Hoai ${ }^{1}$, Pham Tuyet Nhung, Pham Tuan-Anh, Pierre Darriulat, Pham Ngoc Diep, \\ Nguyen Thi Phuong and Tran Thi Thai
}

Department of Astrophysics, Vietnam National Space Center, Vietnam Academy of Sciences and Technologies (VNSC/VAST), 18 Hoang Quoc Viet, Nghia Do, Cau Giay, Hanoi, Vietnam

\begin{abstract}
Millimetre ALMA observations of the nascent winds of several Oxygen rich AGB stars have revealed the high Doppler velocity wings in their spectra. However, the physics underlying their production is unclear. In this paper, we illustrate the argument with four examples of oxygen-rich AGB stars: EP Aqr, R Dor, L2 Pup and Mira Ceti.
\end{abstract}

\section{Introduction}

At the end of their life, when they have burned enough hydrogen into helium to produce a helium core in their centre, stars similar to our Sun blow up by some two orders of magnitude to become Red Giants. They then enter the Asymptotic Giant Branch (AGB) where they start losing mass in the form of a wind of gas and dust. At the same time nuclear reactions take place in the core and new elements are dredged up into the external layers of the star.

The expulsion of material in the interstellar medium is believed to proceed as follows: close to the stellar surface dust grains condense as soon as the temperature is low enough $\left(<10^{3} \mathrm{~K}\right.$ or so). They get radial momentum by absorbing UV radiation from the star, which they transfer to gas molecules by collisions, enough to escape the star gravity. Dust formation depends very much on the $\mathrm{C} / \mathrm{O}$ ratio in the gas atmosphere; most $\mathrm{C}$ and $\mathrm{O}$ atoms bind to produce $\mathrm{CO}$ molecules and whatever is left in excess defines whether one deals with an "oxygen star" or a "carbon star". Carbon-based grains are more effective than oxygen-based grains at absorbing the stellar radiation.

Dust is observed from black body radiation of grains at visible and infrared wavelengths either from space or from ground using adaptative optics and/or interferometry. Gas is best observed from the millimetre emission of $\mathrm{CO}$ molecules that are abundant and easily excited. Observations at millimetre wavelength provide information of the intensity projected on the sky plane and the Doppler velocity. Studies of the morpho-kinematics of O-rich AGB stars show that the gas wind reaches typically a terminal velocity of $\sim 10 \mathrm{~km}$ $\mathrm{s}^{-1}$ at a distance of few hundreds au. However, faint higher velocity wings, in particular in $\mathrm{SiO}$ line profiles, have been observed on many of them.

1 Corresponding author: dthoai@vnsc.org.vn 
The occasional presence of high velocity wings in single dish millimetre observations of the $\mathrm{SiO}$ line emission has been noticed for some time and has been analysed by Winters et al. [1] and more recently by De Vicente et al. [2]. They quote velocities as large as twice the terminal velocity and suggest that the emission occurs close to the star, where $\mathrm{SiO}$ grains have not yet fully formed, and is somehow related to star pulsations.

Recently, the availability of high angular resolution ALMA (Atacama Large Millimeter/sub millimeter Array) observations of several oxygen rich AGB stars like EP Aqr, R Dor, $L_{2}$ Pup and Mira Ceti have shed a new light on this issue [3-6]. However, the origin of these high velocities is still unclear. While EP Aqr seems to favour a scenario in terms of bipolar streams, $\mathrm{L}_{2}$ Pup is naturally described as very high rotation velocities close to the star, while R Dor and Mira Ceti require different interpretations. In this paper, we summarise the state of the art of such studies.

\section{EP Aqr}

EP Aqr is an oxygen-rich star on the early phase of the AGB with no technetium in its spectrum [7] at a distance of $114 \pm 8 \mathrm{pc}$ from the Sun [8-9]. It has a mass between 1 and 2

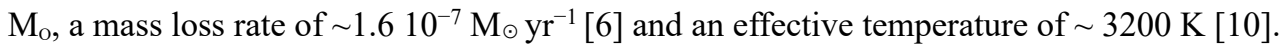

The line emission of different molecules has been observed, among which $\mathrm{CO}, \mathrm{SiO}$ and $\mathrm{SO}_{2}$ which probe preferentially different distances from the star. While $\mathrm{CO}$ molecules are formed early and survive up to long distances, being only affected by UV dissociation from the interstellar medium [11-12], SiO molecules tend to aggregate in grains, causing their progressive disappearance from the gas [13-14] at distances at 100 au scale in addition to their early dissociation from stellar UV radiation [15]. While both $\mathrm{CO}$ and $\mathrm{SiO}$ emissions are excited by collisions with other molecules, in majority hydrogen, $\mathrm{SO}_{2}$ emission is mostly excited (but also dissociated) by absorbing the UV radiation of the star, confining the emission to its close neighbourhood [16-17]. Figure 1 shows the dependence on projected distance to the star, $R$, of the intensity multiplied by $R$. The beam sizes of these observations are $0.33 \times 0.30 \operatorname{arcsec}^{2}, 0.29 \times 0.25 \operatorname{arcsec}^{2}$ and $0.18 \times 0.17 \operatorname{arcsec}^{2}$ for ${ }^{12} \mathrm{CO}(2-1)$, $\mathrm{SiO}(5-4)$ and $\mathrm{SO}_{2}\left(16_{6,10}-17_{5,13}\right)$ respectively.
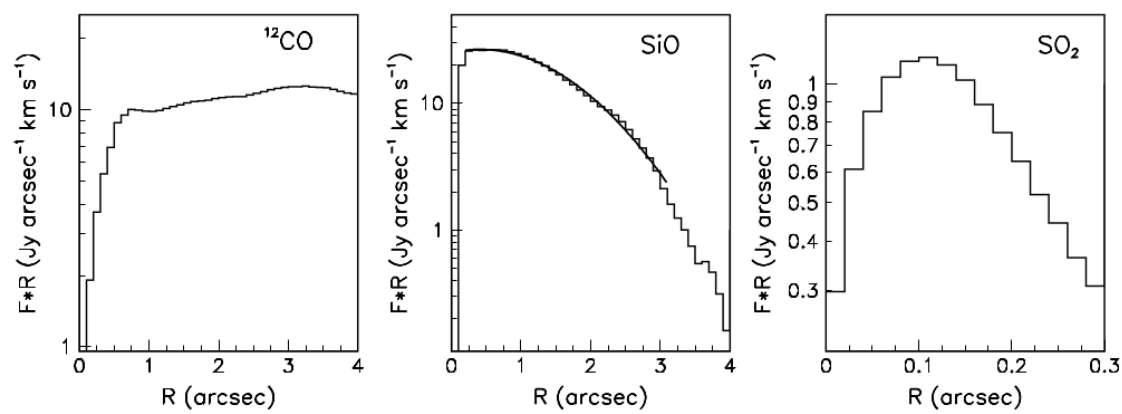

Figure 1: EP Aqr: Dependence on $R$ of the intensity multiplied by $R$ for $\mathrm{CO}, \mathrm{SiO}$ and $\mathrm{SO}_{2}$ (from left to right).

The CO emission at distances exceeding $\sim 250$ au [6] displays approximate axisymmetry about an axis making an angle $\varphi \sim 10^{\circ}$ with the line of sight and projecting on the sky plane some $20^{\circ}$ west of north. The Doppler velocity spectra are made of two components: a narrow component associated with an equatorial outflow expanding at low velocity $V_{e q}=(0.33 \pm 0.03) / \sin \varphi \mathrm{km} \mathrm{s}^{-1}$ and a broad component associated with a bipolar 
outflow expanding at a velocity increasing with stellar latitude $\alpha$ up to 10 to $12 \mathrm{~km} \mathrm{~s}^{-1}$ near the poles. A possible rotation velocity at the equator is shown not to exceed a third of the expanding velocity.

$\mathrm{SiO}$ emission gives evidence for a high velocity $\left(\sim 20 \mathrm{~km} \mathrm{~s}^{-1}\right)$ wind close to the star. The $\mathrm{P}-\mathrm{V}$ maps of Figures 2 show that the wind at short distances reaches a velocity well above the terminal velocity of $\sim 12 \mathrm{~km} \mathrm{~s}^{-1}$ observed in $\mathrm{CO}$ emission at large distances from the star [6].
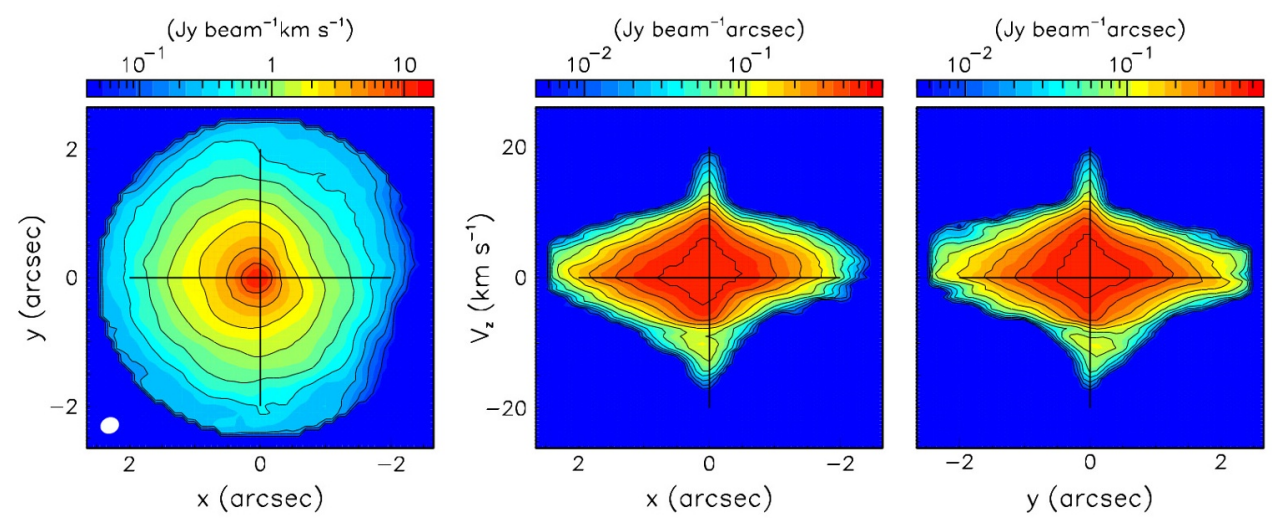

Figure 2: EP Aqr: Projections of the $\mathrm{SiO}$ data-cube on $(x, y)$ (left), $\left(x, V_{z}\right)$ (middle) and $\left(y, V_{z}\right)$ (right).

$\mathrm{SO}_{2}$ emission does not show any evidence for such high velocity components. Instead, the map of the mean Doppler velocity of $\mathrm{SO}_{2}$ (Figure 3) suggests the presence of rotation. Distributions of the Doppler velocity as a function of position angle $\psi$ measured counterclockwise from the $y$ axis (Figure 3 right) is well described by a sine wave; its phase shift nearly cancels, meaning that the axis of rotation projects on the sky plane along the $y$ axis, implying the absence of a significant anisotropy, polar or equatorial, of the expansion velocity [18].
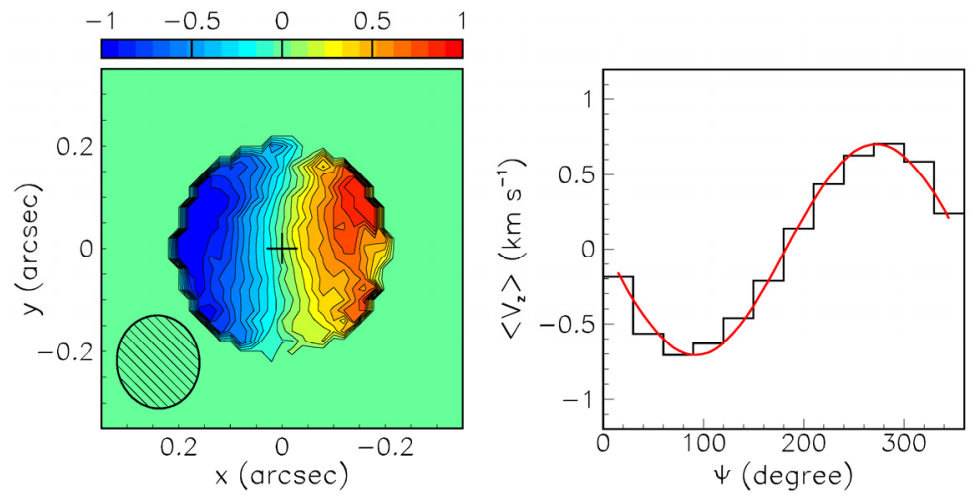

Figure 3: $\mathrm{SO}_{2}$ emission of EP Aqr: sky map of the mean Doppler velocity $\left(\mathrm{km} \mathrm{s}^{-1}, R<0.2 \operatorname{arcsec}\right)$ (left) and dependence of the Doppler velocity on position angle $\psi$ for $0.05<R<0.15 \operatorname{arcsec}$ (right).

The absence of high velocities in $\mathrm{SO}_{2}$ emission, if not the result of insufficient sensitivity, implies that the high velocity wings do not occur very close to the star. It also disfavours an interpretation in terms of a spherical shell ejected by star pulsation at short distances from the star, as described in Winters et al. [1] and McDonald \& Zijlstra [19]. It 
favours instead an interpretation in terms of polar gas streams which is also consistent with the observations at large distances of $\mathrm{CO}$ emission which display clear bipolarity with a factor of 6 between polar and equatorial winds [6].
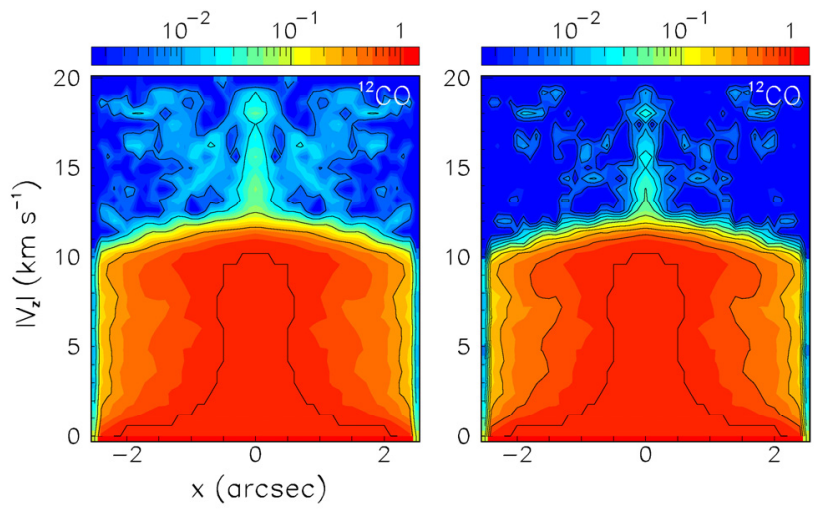

Figure 4: $\mathrm{P}-\mathrm{V}$ maps of the intensity $\left(\mathrm{Jy} \operatorname{arcsec}^{-1}\right)$ for centrally symmetrized $\mathrm{CO}$ observations for a $1.5 \sigma$ noise cut (left) and for a $2 \sigma$ cut (right).

The high velocity streams are present in $\mathrm{CO}$ emission but weaker than in $\mathrm{SiO}$ emission (Figure 4), suggesting that they slow down and/or diverge at large distances from the star. These streams are very narrow $\left(\sim 10^{\circ}\right)$, launched from less than 25 au from the star on the line of sight direction and build up between $\sim 20$ au and $\sim 100$ au to a velocity of $\sim 20 \mathrm{~km}$ $\mathrm{s}^{-1}$ [3]. The mechanism governing the launching of the observed streams remains unknown. However, the coincidence of the stream axis with the symmetry axis of the circumstellar envelope and the $\mathrm{SO}_{2}$ rotation axis strengthens the interpretation of high Doppler velocity wings in terms of gas streams.

\section{L2 Pup}

$\mathrm{L}_{2}$ Puppis is one of the nearest AGB stars at a distance of $64 \pm 4 \mathrm{pc}$ [20]. It is a semi-regular pulsating variable $(\mathrm{P} \sim 140$ days, [21-22] $)$. An asymmetric resolved environment around $\mathrm{L}_{2}$ Pup was first identified by Ireland et al. [23] using aperture masking in the optical and nearinfrared. An edge-on disk with an inclination angle of $82^{\circ}$ has been observed by Kervella et al. [24] in 2014 using VLT/NACO adaptive optics between 1.0 and $4.0 \mu \mathrm{m}$. It was later confirmed by Lykou et al. [25-26], Ohnaka et al. [27] and Kervella et al. [28] in 2015. Kervella et al. [28] identified the inner rim of the disk through its polarimetric signature at a radius of $6 \mathrm{au}$ from the AGB star. They revealed a close companion at a projected separation of 2 au from the main star in R and V band VLT observations, detected bipolar "hourglass" cones in $\mathrm{L}_{2}$ Pup's envelope and discovered streamers in the bipolar cones and two thin, tightly collimated plumes.

The companion is also detected from the continuum and molecular line emissions of ALMA band 7 observations [28]. It has a mass of $12 \pm 16 \mathrm{M}_{\text {Jup }}$ and is most likely a planet or a low-mass brown dwarf with an orbital period of about five years.

In contrast with EP Aqr, $\mathrm{L}_{2}$ Pup displays strong rotation around an axis in the plane of the sky ([4], Figure 5 and 6). It is approximately Keplerian within the central cavity of the dust disk. This allows for estimating the mass of the main star as $\sim 0.66$ solar masses. The nature of the rotation also implies very high rotation velocities close to the star, $\sim 20 \mathrm{~km} \mathrm{~s}^{-1}$ at $1.3 \mathrm{au}$, that appear in projection close to the line of sight. It is therefore natural to interpret the origin of the high Doppler velocities observed close to the star in terms of rotation. 

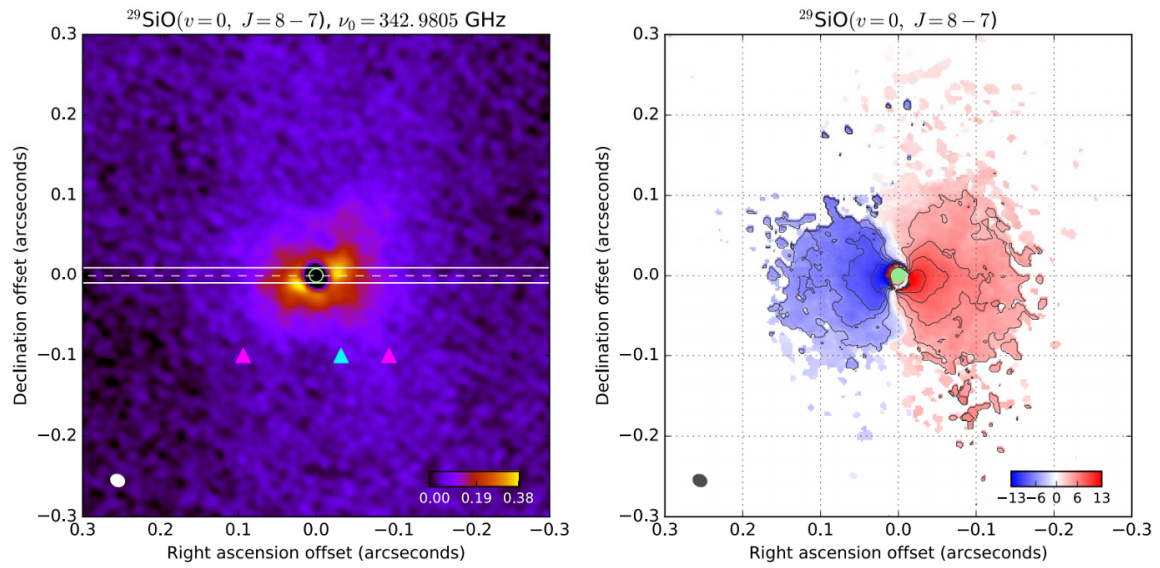

Figure 5: Left panel: map of the emission from $\mathrm{L}_{2} \mathrm{Pup}$ in the ${ }^{29} \mathrm{SiO}(v=0, \mathrm{~J}=8-7)$ line. The solid white lines present the slit of 20 mas used to compute the position-velocity diagrams. Right panel: Doppler velocity map (the colour scale is in $\mathrm{km} \mathrm{s}^{-1}$ ).
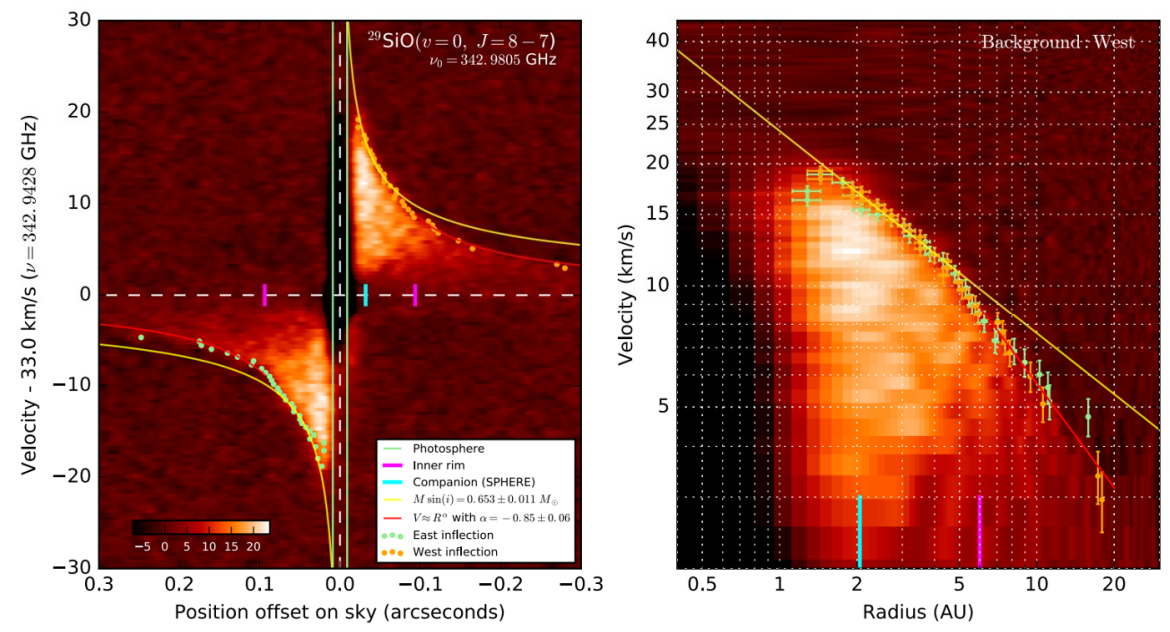

Figure 6: $\mathrm{L}_{2}$ Pup: Position-velocity diagrams plotted inside the slit shown in Figure 5. The yellow curve is a Keplerian fit to the inner cavity, and the red curve is a power law fit to the non-Keplerian radius domain.

\section{R Dor}

R Dor is an oxygen rich AGB star very similar to EP Aqr in terms of spectral classes, masses, mass loss rates and temperatures. It also has no technetium in its spectrum [30]. It is the closest AGB star to the Sun, at distance of $\sim 59 \mathrm{pc}$ [29]. It has a dual pulsation period of 175 and 332 days [30].

The circumstellar envelope of R Dor has been probed by ALMA with a resolution of $\sim 4$ au up to some $60 \mathrm{au}$ from the star [31-33] and the dust has been observed at the Very Large Telescope (VLT) with a resolution of 1.2 au [34]. In addition, below $\sim 15$ au, the analyses of Danilovich et al. [16], De Beck \& Olofsson [35] and Van de Sande et al. [14] have contributed a considerable amount of detailed information of relevance to the physico- 
chemistry and dynamics of both dust and gas. At larger distances from the star, an analysis of ALMA observations of $\mathrm{SO}\left(\mathrm{J}_{\mathrm{K}}=6_{5}-5_{4}\right)$ emission [36], probing distances between 20 and $100 \mathrm{au}$, gives evidence for the wind to host a radial outflow covering large solid angles and displaying strong inhomogeneity both in direction and radially: the former takes the form of multiple cores and the latter displays a radial dependence suggesting an episode of enhanced mass loss having occurred a century or so ago.

Decin et al. [31] were first to discuss the presence of high velocity wings, far above the canonical terminal wind velocities, not only in the $\mathrm{SiO}$ but also in the $\mathrm{CO}$ line emissions from the vibrational ground state, with too low an excitation energy to be caused by pulsations [37]. They argue that neither star pulsations nor thermal motions of the gas can significantly contribute to the generation of such high velocities.

Hoai et al. [5] made a more detailed and dedicated study of this issue; they use line emissions detected between $\sim 15$ and $\sim 50$ au from the star, including five different molecular excitations: $\mathrm{CO}(3-2), \mathrm{SiO}(8-7), \mathrm{SO}\left(6_{5}-5_{4}\right), \mathrm{SO}_{2}\left(13_{4,10}-13_{3,11}\right)$ and $\mathrm{HCN}(4-3)$ with an angular resolution of $\sim 0.16$ arcsec.
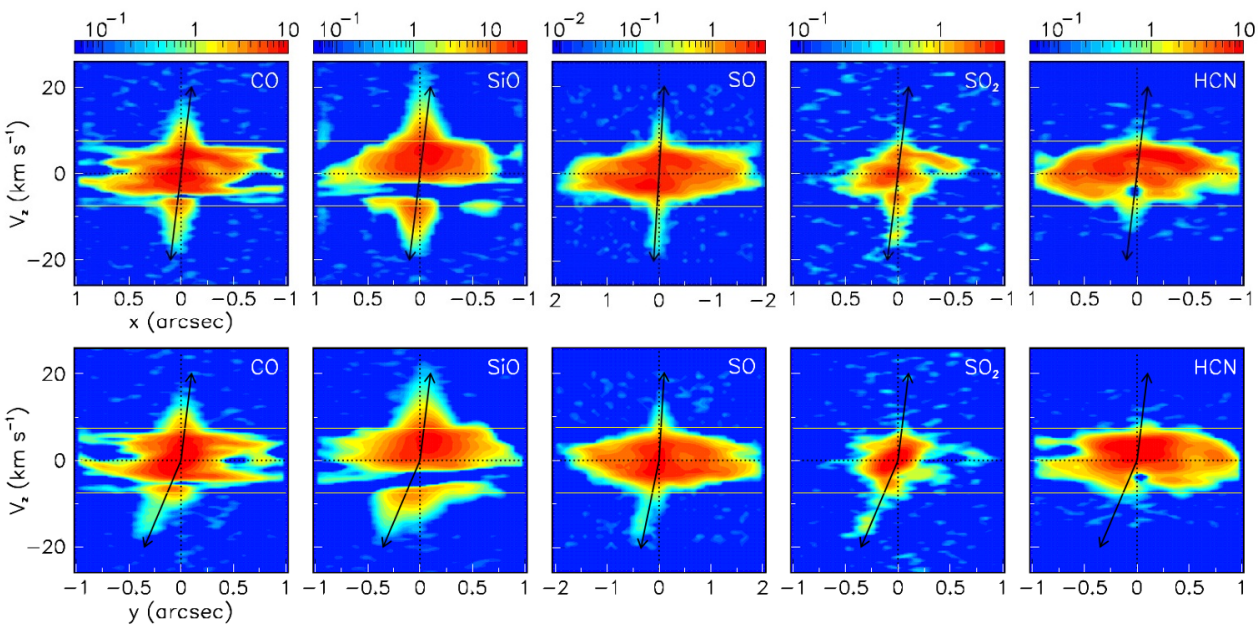

Figure 7: R Dor: P-V maps in the $V_{z}$ vs $x$ and vs $y$ planes. The colour scale is in units of Jy $\operatorname{arcsec}^{-1}$. Yellow lines show the cuts applied in the definition of the large Doppler velocity components.

Projections of the data-cubes on the $\left(x, V_{z}\right)$ and $\left(y, V_{z}\right)$ planes (P-V maps) are shown in Figure 7. They are the integration over $y$, pointing $35^{\circ}$ west of north, and $x$, pointing $35^{\circ}$ north of east, respectively. In all cases, the larger values of $\left|V_{z}\right|$ are confined near $x=0$, very much as was observed in EP Aqr. They are particularly visible in the $\mathrm{CO}, \mathrm{SiO}$ and $\mathrm{SO}$ maps, both in the blue-shifted and red-shifted hemispheres, but more clearly in the former than in the latter.

If these high velocity winds are ejected in the form of gas streams and reach velocity of $20 \mathrm{~km} \mathrm{~s}^{-1}$ at a same distance in space like in the case of EP Aqr, the red-shifted and blueshifted streams should be both confined near the line of sight and nearly back-to-back. However, in variation to EP Aqr, the rotation axis observed by Homan et al. [32] makes an angle of only $20 \pm 20^{\circ}$ with the plane of the sky, far from the line of sight. This may suggest that the observed high Doppler velocity wings are artefacts of improper data reduction and/or continuum subtraction; however, the Doppler velocity distribution of continuumunsubstracted data (Figure 8) shows that such is not the case: the high velocity wings are still present in continuum-unsubstracted data. Blue-shifted and red-shifted high velocity components display amazing similarity in spite of some expected absorption. 

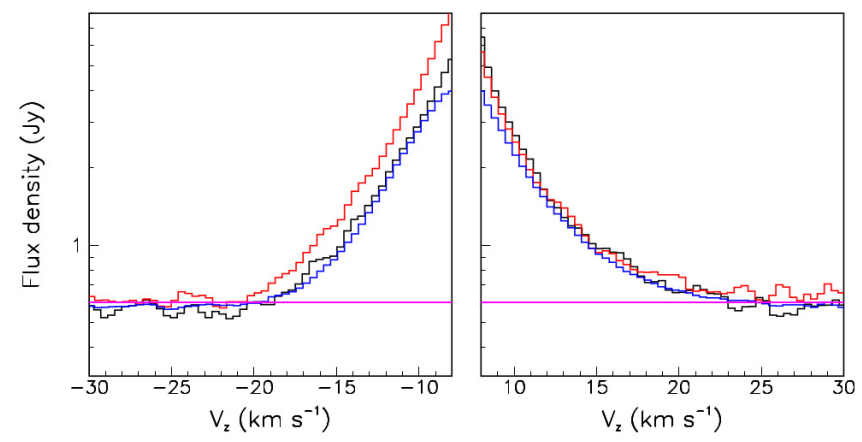

Figure 8: Doppler velocity distributions obtained for $\left|V_{z}\right|>8 \mathrm{~km} \mathrm{~s}^{-1}$ using two data sets of SiO line emission having significantly different uv coverage. The data set having the larger maximal recoverable scale is shown for $R<1.5 \operatorname{arcsec}$ (red) and for $R<0.3 \operatorname{arcsec}$ (blue). The data set having the smaller maximal recoverable scale is shown for $R<1.5 \operatorname{arcsec}$ (black).

Interpretation in terms of rotation is difficult to justify in this case. Homan et al. [32] quote a rotation velocity of only $12 \mathrm{~km} \mathrm{~s}^{-1}$ at a distance of 6 au from the star. Keplerian rotation would imply that the maximal observed velocity of $20 \mathrm{~km} \mathrm{~s}^{-1}$ would be reached at only $\sim 2.2$ au from the star centre, very close to the stellar surface. The absence of detection in the ${ }^{28} \mathrm{SiO}(v=1, \mathrm{~J}=8-7)$ data analyzed by Homan et al. [32] would then have to be blamed on insufficient sensitivity if a Keplerian rotation scenario were retained. Vlemmings et al. [33] quote a solid body rotation of $1.0 \pm 0.1 \mathrm{~km} \mathrm{~s}^{-1}$ at the stellar surface $(\sim 1.9 \mathrm{au})$ meaning that a rotation velocity of $20 \mathrm{~km} \mathrm{~s}^{-1}$ would be reached at a distance of $\sim 38$ au from the star, nearly twice as far as the canonical limit that has been set. Unfortunately, the authors do not comment on the high Doppler velocity wings in general. They only illustrate the blueshifted stream, which they claim to be one-sided, with a $\mathrm{P}-\mathrm{V}$ diagram of ${ }^{29} \mathrm{SiO}(v=0, \mathrm{~J}=5-4)$ data that is consistent with the gas stream interpretation. However, both rotation and pulsation predict red-blue symmetry in contrast with what is observed.

\section{Mira Ceti}

Mira Ceti is one of the most studied AGB stars, close to the Sun $\sim 100 \pm 10$ pc $[9,38]$. It is remarkable for the large amplitude of its variability, $\sim 8 \mathrm{mag}$, with a periodicity of $\sim 333$ days [39]. The direct evidence for a companion was first obtained in 1995 by the Hubble Space Telescope in visible light [40]. Its orbit is poorly constrained by observations [41]: if circular its projected eccentricity corresponds to an angle of $\sim 22^{\circ}$ with the line of sight, the semi-major axis being $\sim 0.8$ arcsec. The orbital period is estimated to be at least 500 years. While the companion has been resolved at many other wavelengths, its nature is still a matter of debate, some claiming that it is a White Dwarf [42-44] and others claiming that it is a Main Sequence star [45].

Recent studies using high resolution observations of ALMA at radio wavelengths have shown the complex morpho-kinematics of the circumstellar envelope of Mira AB [6,46-47].

Close to the stars $\left(R<0.25\right.$ arcsec), large values of $\left|V_{z}\right|$ reaching $16 \mathrm{~km} \mathrm{~s}^{-1}$ for both CO and $\mathrm{SiO}$ have been revealed (Figure 9 ). They are more clearly marked when centred on the main star, Mira A, than on its companion, Mira B. This observation raises the question of the origin of such large Doppler velocity wings.

Since there is no evidence for the line of sight to play a particular role in the dynamics of the Mira AB pair, it would be unreasonable to retain an interpretation in terms of gas 
streams. Moreover no significant rotation is detected near Mira A, leaving stellar pulsation as a candidate interpretation.

However, $\mathrm{CO}$ and $\mathrm{SiO}$ observations have been made at very different phases of the Mira pulsation cycle [48-49], $\mathrm{CO}$ near maximum and $\mathrm{SiO}$ near minimum. The radial velocity of the stellar pulsation is expected to span some 20 to $30 \mathrm{~km} \mathrm{~s}^{-1}$ between maximum and minimum [37]: the velocities measured in $\mathrm{CO}$ and $\mathrm{SiO}$ emissions should be different, in contradiction with observations.
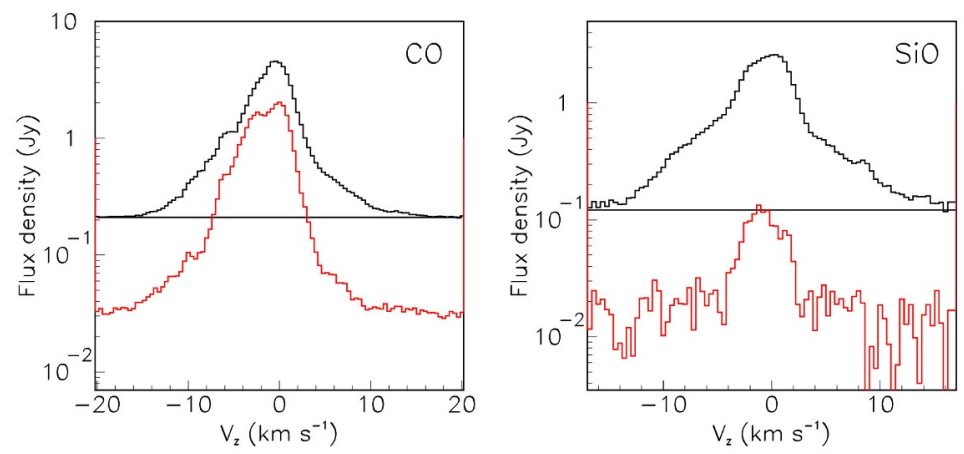

Figure 9: Mira Ceti: Doppler velocity distributions of CO (left) and SiO (right) line emission within a circle of 0.25 arcsec projected radius centred on the main star Mira A (black, upper) or its companion Mira B (red, lower).
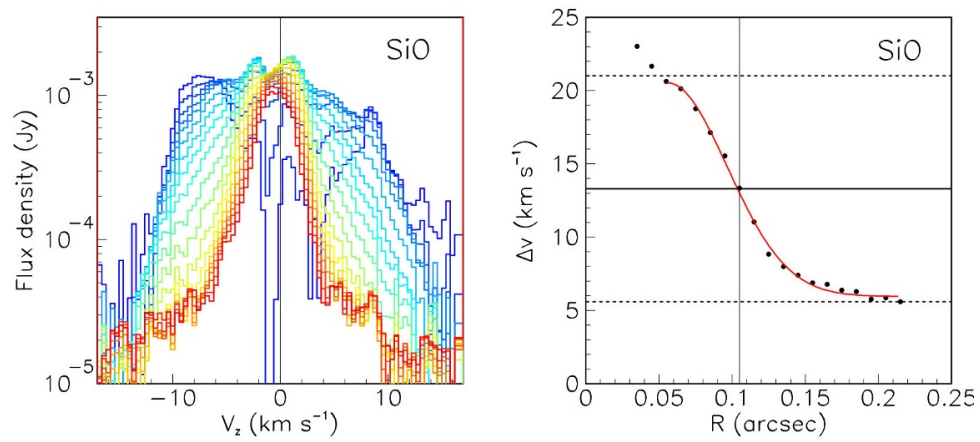

Figure 10: Mira Ceti: Left: Doppler velocity spectra of $\mathrm{SiO}$ averaged in 0.03 arcsec wide rings centred on Mira A with mean radii increasing from $0.025 \operatorname{arcsec}$ (blue) to $0.215 \operatorname{arcsec}$ (red) in steps of 0.01 arcsec. Right: dependence on $R$ of the full-with at 1/5 maximum of the spectra displayed in the centre-right panel.

The spectra of $\mathrm{SiO}$ in different rings centred on the star (Figure 10) are broader close to the star and narrower at larger distances with a rather sharp transition at $\sim 0.1$ arcsec. It suggests that these large velocities are reached at typical distances of 5 to 10 au from Mira A and are caused by a large line width rather than by a large wind velocity. As thermal broadening cannot produce such large line widths, turbulence must be invoked. The picture would then be of a shell surrounding the zone where stellar pulsation dominates and extending up to $\sim 10 \mathrm{au}$, hosting a very turbulent regime. Indeed, such a possibility was mentioned earlier by Kamínski et al. [50] in relation with the possible presence of very broad AlO line profiles. According to the standard picture of acceleration by transfer of momentum from dust grains to molecules of gas([51], this is a region that hosts important shocks. At $10 \mathrm{au}$, the escape velocity from one (two) solar masses, values commonly accepted for Mira A, is $\sim 13$ (19) $\mathrm{km} \mathrm{s}^{-1}$ and the density is such that the velocity acquired by 
a gas molecule after its interaction with a dust grain is immediately thermalized. Such important turbulence has been observed in a different context by Falgarone \& Phillips [52] and discussed by Gillet et al. [53] in the case of pulsating stars.

\section{Summary}

The presence of large Doppler velocities in molecular line emission spectra of oxygen-rich AGB stars has been observed for quite some time but is still not thoroughly understood. We have discussed here the cases of four stars close enough to the Sun to have lent to high angular resolution measurements. However, these observations suggest interpretations that differ from star to star, making one be suspicious of their ad hoc nature. The interpretation as gas streams favoured in the case of EP Aqr seems difficult to extend to the cases of the other stars, for which the line of sight plays no special role. The interpretation in terms of rotation favoured in the case of $\mathrm{L}_{2}$ Pup has been shown not to apply to the other stars. Simple interpretations in terms of stellar pulsations have been shown to be untenable quantitatively. To the extent that such large Doppler velocity wings share many properties, it seems desirable to find a common interpretation that could apply to all cases. The possible importance of turbulence in a layer surrounding the pulsation region revealed by the study of Mira Ceti needs to be better understood. A global analysis of all available observations of relevance, together with additional high angular resolution observations are required to better sort out the arguments of relevance and deepen our understanding of the mechanisms at stake.

We are deeply indebted to the ALMA partnership, whose open access policy means invaluable support and encouragement for Vietnamese astrophysics. Financial support from the World Laboratory, VNSC and NAFOSTED is gratefully acknowledged. This research is funded by the Vietnam National Foundation for Science and Technology Development (NAFOSTED) under grant number 103.99-2018.325.

\section{Reference}

1. Winters J. M., Le Bertre T., Jeong K. S., Nyman L.-Å., Epchtein N., A\&A, 409, 715 (2003)

2. De Vicente P. et al., A\&A, 589, A74 (2016)

3. Tuan-Anh et al., MNRAS, 487, 622 (2019)

4. Kervella P., Homan W. , Richards A. M. S. et al., A\&A, 596, A92 (2016)

5. Hoai et al., Comm. Phys. Vietnam, 20, 85, 3 (2020), aXiv: 1906.08535

6. Hoai D. T. et al., MNRAS, 484, 1865 (2019)

7. Lebzelter, T., and Hron, J., A\&A, 351, 533 (1999)

8. Gaia Collaboration, A\&A, 616, A1 (2018)

9. van Leeuwen F., A\&A, 474, 653 (2007)

10. Dumm T., \& Schild H., New Astr., 3, 137 (1998)

11. Groenewegen M. A. T., A\&A, 606, A67 (2017)

12. Mamon G. A., Glassgold A. E., Huggins P. J., ApJ, 328, 797 (1988)

13. Gonzalez Delgado D., Olofsson H., Kerschbaum F. et al., A\&A, 411, 123 (2003)

14. Van de Sande M., Decin L., Lombaert R. et al., A\&A, 609, A63 (2018)

15. Willacy K., Millar T. J., A\&A, 324, 237 (1997)

16. Danilovich T., De Beck E., Black J. H., Olofsson H., Justtanont K., A\&A, 588, A119 (2016)

17. Yamamura I., de Jong T., Onaka T., Cami J., Waters L. B. F. M., A\&A, 341, L9 (1999)

18. Diep P. N., Phuong N. T., Hoai D. T. et al., MNRAS, 461, 4276 (2016)

19. McDonald I., Zijlstra A. A., ApJ, 823, L38 (2016)

20. van Leeuwen F., A\&A, 474, 653 (2007)

21. Bedding, T. R., Kiss, L. L., Kjeldsen, H., et al., MNRAS, 361, 1375 (2005) 
22. Kholopov, P. N., Samus, N. N., Kazarovets, E. V., \& Perova, N. B., Information Bulletin on Variable Stars, 2681, 1 (1985)

23. Ireland, M. J., Tuthill, P. G., Bedding, et al., MNRAS, 350, 365 (2004)

24. Kervella, P., Montargès, M., Ridgway, S. T. et al., A\&A, 564, A88 (2014)

25. Lykou, F., Klotz, D., Paladini, C. et al., A\&A, 576, A46 (2015)

26. Lykou, F., Klotz, D., Paladini, C. et al., A\&A, 581, C2 (2015)

27. Ohnaka, K., Schertl, D., Hofmann, K.-H. \& Weigelt, G., A\&A, 581, A127 (2015)

28. Kervella, P., Montargès, M., Lagadec, E., et al., A\&A, 578, A77 (2015)

29. Knapp G.R., Pourbaix D., Platais, I., and Jorissen, A., A\&A, 403, 993 (2003)

30. Bedding T. R., Zijlstra A.A., Jones A., \& Foster G., MNRAS, 301, 1073 (1998)

31. Decin L., Richards A.M.S., Danilovich T., et al., A\&A, 615, A28 (2018)

32. Homan W., Danilovich T., Decin L., et al., A\&A, 614, A113 (2018b)

33. Vlemmings W.H.T., Khouri T., De Beck E., et al., A\&A, 613, L4 (2018)

34. Khouri T., Maercker M., Waters L.B.F.M., et al., A\&A, 591, A70 (2016)

35. De Beck E. \& Olofsson H., A\&A, 615, A8 (2018)

36. Nhung P.T., Hoai D.T., Tuan-Anh P., et al., MNRAS, 490, 3329 (2019)

37. Nowotny, W., Höfner, S. and Aringer, B., A\&A, 514, A35 (2010)

38. Haniff, C. A., Scholz, M., \& Tuthill, P. G., MNRAS, 276, 640 (1995)

39. Templeton M. R. \& Karovska M., 2009, ApJ, 691, 1470 (2009)

40. Karovska, M., Hack, W., Raymond, J., \& Guinan, E., ApJ, 482, L175 (1997)

41. Prieur, J. L., Aristidi, E., Lopez, B. et al., ApJS, 139, 249 (2002)

42. Reimers, D. \& Cassatella, A., ApJ, 297, 275 (1985)

43. Wood, B. E. \& Karovska, M., ApJ, 649, 410 (2006)

44. Sokoloski, J. L. \& Bildsten, L., ApJ, 723, 1188 (2010)

45. Ireland, M.J., Monnier, J.D., Tuthill, P.G. et al., ApJ, 662, 651 (2007)

46. Ramstedt, S., Mohamed, S., Vlemmings, W.H.T., et al., A\&A, 570, L14 (2014)

47. Nhung, P.T., Hoai, D.T. Diep, P.N. et al., MNRAS, 460, 673 (2016)

48. Planesas, P., Alcolea, J. \& Bachiller, R., A\&A, 586, A69 (2016)

49. Wong, K.T., Kamínski, T., Menten, K.M. \& Wyrowski, F., A\&A, 590, A127 (2016)

50. Kamínski, T., Wong, K.T., Schmidt, M.R. et al., A\&A, 592, A42 (2016)

51. Höfner, S. \& Olofsson, H., A\&ARev., 26, 1 (2018)

52. Falgarone, E. \& Phillips, T.G., ApJ, 359, 344 (1990) 